\title{
EXPERIMENTAL MODELS OF METABOLIC SYNDROME IN RATS
}

\author{
Silvia Gancheva ${ }^{1}$, Maria Zhelyazkova-Savova ${ }^{1}$, Bistra Galunska ${ }^{2}$, Trifon Chervenkov ${ }^{3}$ \\ ${ }^{1}$ Department of Preclinical and Clinical Pharmacology, Medical University of Varna \\ ${ }^{2}$ Department of Pharmaceutical Technologies, Medical University of Varna \\ ${ }^{3}$ Department of Pediatrics and Medical Genetics, Medical University of Varna
}

\begin{abstract}
Metabolic syndrome (MS) is a disorder comprising central obesity, dyslipidemia, raised blood pressure, insulin resistance. The aim of the present study was to develop a cheap, easy and reproducible rat model of MS. 36 male Wistar rats were divided in 3 groups: a control group (C) receiving regular rat chow diet, a high-fat (HF) group receiving lard enriched rat chow and a high-fat high-fructose (HFHF) group receiving lard and fructose enriched rat chow. HF and HFHF groups had also $10 \%$ fructose in their drinking water. The duration of the study was 8 weeks. Body weights were measured weekly. At the end of the study insulin tolerance test (ITT) was performed. Liver and fat weight index were measured after sacrifice. Lipid biochemical parameters and insulin concentration in serum were determined. Liver triglycerides (TG) were measured. The oxidative stress in serum was assessed by thiobarbituric reactive substances (TBARS). At the end of the study the animals did not differ in their body weights across the groups, but the fat index in both HF and HFHF groups was higher. Plasma TG and cholesterol were raised in both groups and the ratio cholesterol/ HDL-cholesterol was higher. Liver TG were elevated in HFHF rats. ITT revealed reduced insulin sensitivity in both experimental groups although serum insulin was elevated only in HFHF group. TBARS were increased in both HF and HFHF groups. Both models displayed most of the features of MS; the HFHF probably better reflects the 'cafeteria' diet and its unhealthy consequences.
\end{abstract}

Keywords: metabolic syndrome, rat model, high fat diet, high fat high fructose diet

\section{INTRODUCTION}

The metabolic syndrome (MS) is a condition of impaired energy metabolism. About 25\% of the adults worldwide suffer MS according to the International Diabetes Federation (IDF) (1). The most significant factors for the development of MS are considered insulin resistance (the DECODE study) (2)

Address for correspondence:

Silvia Gancheva, $M D, P h D$

Department of Preclinical and Clinical Pharmacology,

Medical University of Varna

55 Marin Drinov Str.,

9002 Varna, Bulgaria

e-mail: silvi_gancheva@abv.bg

Received: April 04, 2015

Accepted: May 28, 2015 and the visceral obesity (3). IDF defines MS as a condition including visceral obesity and at least two of the following: high triglycerides, low HDL-cholesterol, high blood pressure, and fasting hyperglycemia as a result of insulin resistance (1).

The experimental models of MS are valuable tools in the study of its pathogenesis, prevention and therapy. There are many such models in rodents and other experimental animals, but no one has been universally accepted. Since non-rational nutrition with high caloric intake is considered one of the leading causes for MS in humans (4), the animal models frequently rely on manipulating the dietary intake. The diets used to develop models of MS contain high amounts of carbohydrates, fats or both. Fructose is the preferred monosaccharide, included in the ani- 
Silvia Gancheva, Maria Zhelyazkova-Savova, Bistra Galunska et al.

mal food or drinking water. One of the most popular models is the one with $10 \%$ fructose solution as a drinking water given over several weeks $(5,6,7)$. Another approach is to add different fats to the food. Animal fats turn out to be more effective to produce signs of MS compared to plant-derived fats $(8,9)$. Lard or beef fat are usually used. The mixed models utilizing both animal fats rich in saturated fatty acids and fructose are considered most relevant to the MS in men $(10,11,12,13)$.

THE AIM of the present study was to develop an easy, cheap and reproducible experimental model of MS, displaying most of the characteristic features of the condition in men. We have studied and characterized two models of MS in rats with increased content of simple carbohydrates and fats in their diet, given in different ratios.

\section{MATERIALS AND METHODS \\ Experimental animals}

The study has been performed on 36 male Wistar rats. The animals were kept at an ambient temperature of $20-25^{\circ} \mathrm{C}, 12$-hour light-dark cycle and free access to food and water. The study has been approved by the Commission of foods safety in the Ministry of Agriculture and Foods.

The experimental animals were allocated in 3 groups of 12 rats each, with initial body weight ranging between 204 and $232 \mathrm{~g}$ (on the average, 219-220 $\mathrm{g}$ in each group). The duration of the study was 8 weeks. The groups were as follows: a control group (C), a group fed high fat (HF) diet and a group fed high fat and high fructose (HFHF) diet.

\section{Diets}

The rats from the control group were fed by the standard rat chow and were given plain water to drink. With each $100 \mathrm{~g}$ food consumed, the animals from this group had a caloric intake of $279 \mathrm{kcal}$.

The rats from the HF group had a diet rich in lard $(20 \%)$ and drank $10 \%$ fructose solution. In this way HF group had a caloric intake of $403 \mathrm{kcal}$ per $100 \mathrm{~g}$ food, of which $45 \%$ was coming from the fat added. These rats also consumed $10 \%$ fructose in the drinking water, allowing additional $40 \mathrm{kcal}$ per 100 $\mathrm{ml}$ water consumed.

The rats from the third group (HFHF) were given a diet with lard (17\%) and fructose (17\%) added to the standard rat chow together with $10 \%$ of fructose in the drinking water. The caloric intake was 405 kcal per $100 \mathrm{~g}$ food, of which $38 \%$ was provided by the lard and $17 \%$ by the fructose added to the standard rat chow. These animals had also additional 40

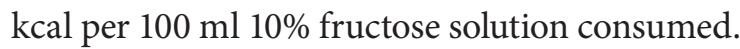

The food and fluids consumption was measured daily. The body weight was taken once weekly.

\section{Insulin tolerance test (ITT)}

After 8 weeks of a diet load an insulin tolerance test was carried out. The animals were injected i.p. with regular insulin (ActRapid), dissolved in saline, at a dose of $0.75 \mathrm{UI} / \mathrm{kg}$. Blood sugar was measured by a glucometer (ACCU-CHEK Performa). Blood samples were taken by incision of the distal part of the tail (14) immediately before the injection of insulin (at time 0 ) and at the $30^{\text {th }}, 60^{\text {th }}$ and $90^{\text {th }}$ minute. Blood was taken from non-fasted animals.

At the end of the experimental period blood was taken from the sublingual veins of the animals under ether anesthesia. The blood was centrifuged and the serum was refrigerated at $-20^{\circ} \mathrm{C}$ until biochemically tested.

After euthanasia of the rats the livers and the right retroperitoneal fat pads were dissected on ice, measured and the corresponding organ indices calculated (as a ratio to the body weight $\times 10^{3}$ ). A part of the liver was stored at $-20^{\circ} \mathrm{C}$ for biochemical testing.

\section{Biochemical tests}

Serum triglycerides (TG), cholesterol and HDLcholesterol were measured by using colorimetric kits of HUMAN, at a spectrophotometer AURIUS 2021 (Cecil Instruments Ltd.). TGs in the liver were determined in $10 \%$ tissue homogenates in TRIS buffer. The atherogenic ratios cholesterol/HDL-cholesterol and TG/HDL-cholesterol were also calculated $(15,16)$.

The levels of serum insulin were measured by ELISA (Rat Insulin ELISA Kit of Shibayagi Co., Ltd.).

As markers of oxidative stress in serum, substances reacting with thiobarbituric acid (TBARS) were measured by a colorimetric method (17) with malondialdehyde as a standard.

\section{Statistics}

Results are presented as a mean \pm standard error of the mean (SEM). Two groups were compared by Student's t-test. Differences were considered sig- 
nificant at $\mathrm{p}<0.05$. The statistical software GraphPad Prism 5 was used (GraphPad Software, Inc.).

\section{RESULTS}

Experimental groups consumed significantly less food than the control animals and HFHF animals consumed more food than HF animals. At the same time experimental groups consumed more fluids than the control group and the HFHF animals also drank more $10 \%$ fructose compared to the HF group. All groups differed in their total daily caloric intake from food and drinking water (Table 1).

At the end of the study body weight of the animals did not differ among groups. There was no difference between groups in respect to liver weights

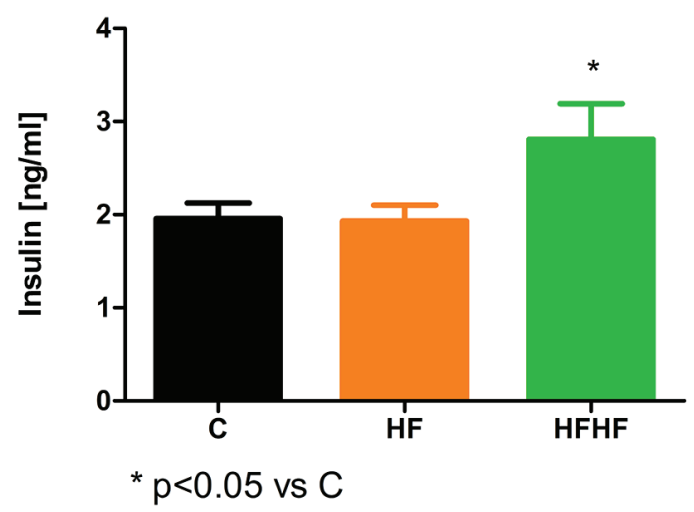

Fig. 2. Serum insulin levels

Table 1. Biological parameters of rats from the control (C), high fat (HF) and high fat high fructose (HFHF) group; ** $p<0.01$, ${ }^{* *} p<0.001$ vs $C$; \# $p<0.05$, \#\# $p<0.001$ vs HF

\begin{tabular}{|c|c|c|c|}
\hline & C & $\mathrm{HF}$ & HFHF \\
\hline Food intake (g/day/group) & $149.2 \pm 1.42$ & $89.97 \pm 1.75^{\star \star \star}$ & $91.86 \pm 1.67^{\star \star \star \#}$ \\
\hline Fluid intake (ml/day/group) & $199.3 \pm 2.38$ & $240 \pm 3.31^{* * *}$ & $273.2 \pm 5.56^{\star * *} \# \# \#$ \\
\hline Caloric intake (kcal/day/group) & $416.9 \pm 3.92$ & $446.4 \pm 6.97^{\star * *}$ & 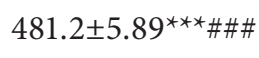 \\
\hline Initial body weight (g) & $219.2 \pm 2.14$ & $220 \pm 1.23$ & $219.5 \pm 2.12$ \\
\hline Final body weight $(\mathrm{g})$ & $348.8 \pm 8.35$ & $351.5 \pm 8.75$ & $360.8 \pm 6.34$ \\
\hline Liver weight (g) & $14.16 \pm 0.69$ & $15.22 \pm 0.85$ & $15.26 \pm 0.41$ \\
\hline Liver index & $4.03 \pm 0.14$ & $4.2 \pm 0.15$ & $4.18 \pm 0.14$ \\
\hline Fat weight $(\mathrm{g})$ & $2.31 \pm 0.26$ & $4.43 \pm 0.45^{\star *}$ & $5.51 \pm 0.75^{\star *}$ \\
\hline Fat index & $6.61 \pm 0.75$ & $12.18 \pm 1.04^{\star *}$ & $15 \pm 1.85^{\star *}$ \\
\hline
\end{tabular}

and liver index. The retroperitoneal fat was significantly increased in HF and HFHF groups compared to controls. Biological parameters are presented on Table 1.

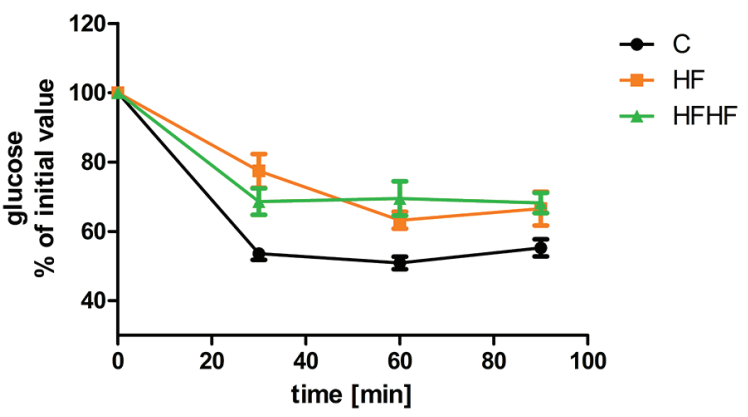

Fig. 1. Insulin tolerance test
The ITT showed impaired response to insulin in both HF and HFHF group. The results are presented on Fig. 1 and Table 2.

The level of serum insulin was elevated in the group HFHF (Fig. 2).

\section{Serum lipids}

TGs and cholesterol in serum were increased in both dietary manipulated groups compared to the control. HDL-cholesterol showed no change. Lipid profile is presented on Fig. 3. Atherogenic indices were higher in rats from the HF and HFHF groups (Fig. 4).

The liver TGs were increased in HFHF relative to the control and HF groups (Fig. 5). 
Silvia Gancheva, Maria Zhelyazkova-Savova, Bistra Galunska et al.

Table 2. Plasma glucose concentration in insulin tolerance test (ITT) in rats from the control (C), high fat (HF) and high fat high fructose (HFHF) group; ${ }^{*} p<0.05,{ }^{* *} p<0.01,{ }^{* * *} p<0.001$ vs $C$

\begin{tabular}{|l|c|c|c|c|c|c|}
\hline \multicolumn{5}{|c|}{$30 \mathrm{~min}$} & \multicolumn{2}{c|}{$90 \mathrm{~min}$} \\
\hline & $\mathrm{mmol} / \mathrm{L}$ & $\%$ of initial value & $\mathrm{mmol} / \mathrm{L}$ & \% of initial value & $\mathrm{mmol} / \mathrm{L}$ & \% of initial value \\
\hline C & $3.64 \pm 0.12$ & $53.56 \pm 1.76$ & $3.46 \pm 0.11$ & $50.89 \pm 1.81$ & $3.74 \pm 0.15$ & $55.22 \pm 2.48$ \\
\hline HF & $4.31 \pm 0.23$ & $71.3 \pm 3.11^{* *}$ & $3.77 \pm 0.17$ & $63.22 \pm 2.41^{* * *}$ & $3.93 \pm 0.27$ & $66.56 \pm 4.87$ \\
\hline HFHF & $4.22 \pm 0.17^{*}$ & $65.73 \pm 2.75^{* *}$ & $4.18 \pm 0.15^{* *}$ & $65.45 \pm 3.13^{* *}$ & $4.37 \pm 0.19^{*}$ & $68.2 \pm 2.92^{* *}$ \\
\hline
\end{tabular}

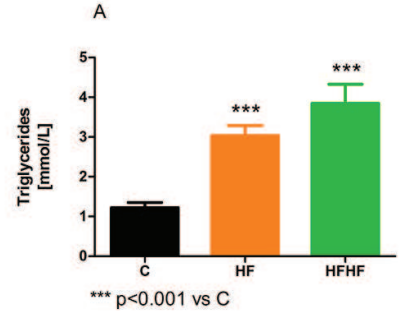

A

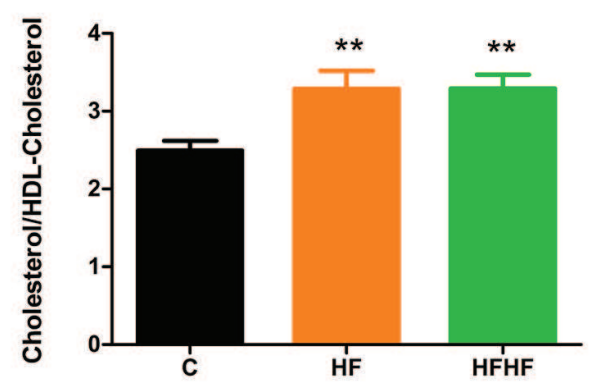

${ }^{* *} p<0.01$ vs $C$
B

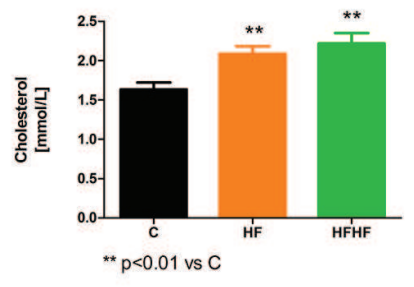

Fig. 3. Serum lipid profile
C

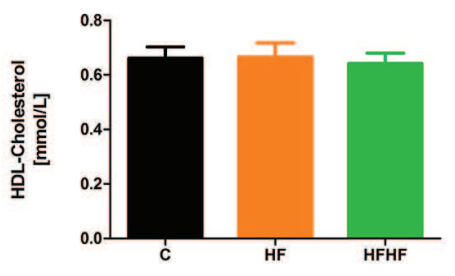

B

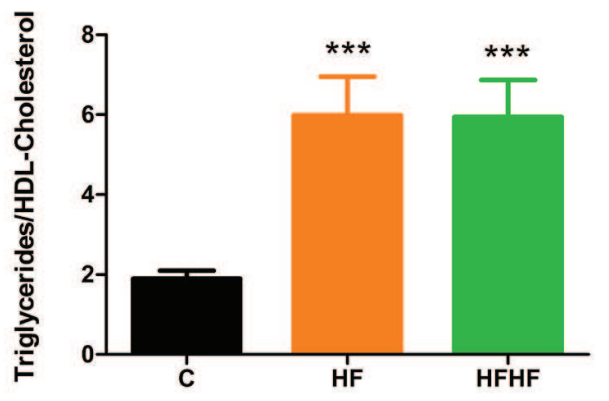

*** $p<0.001$ vs $C$

Fig. 4. Atherogenic indices

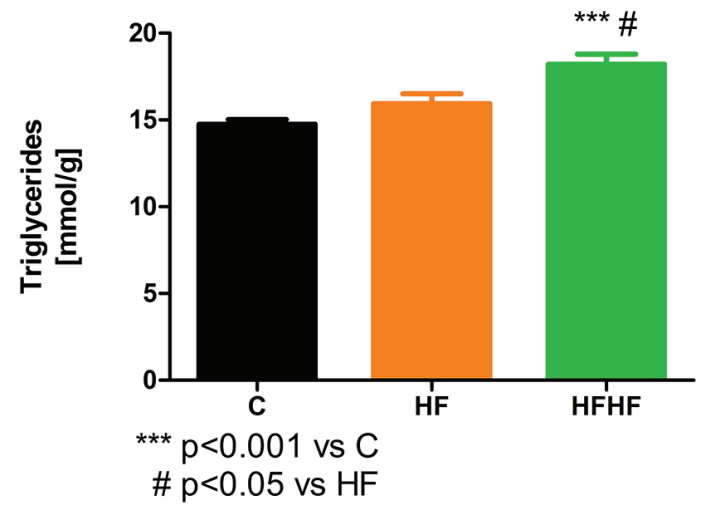

Fig. 5. Liver triglycerides

The serum concentration of TBARS was increased relative to control rats in both HF and HFHF groups (Fig. 6).

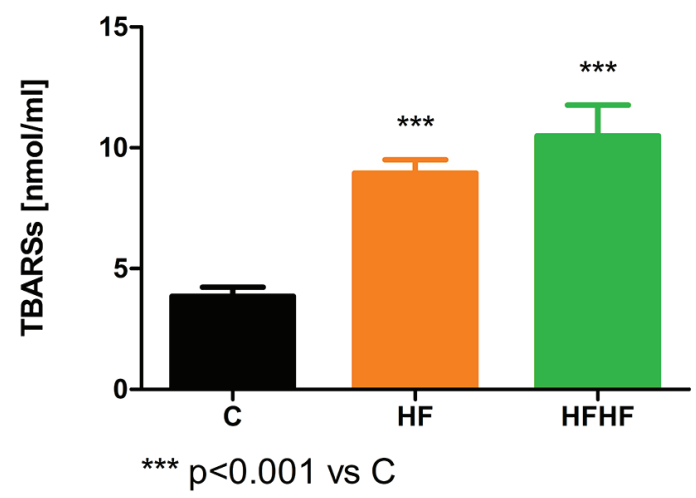

Fig. 6. Concentration of TBARSs in serum

\section{DISCUSSION}

The experimental models of MS aim at reproducing most of the characteristic features of the syn- 
drome in humans, such as overweight, visceral obesity, dyslipidemia, insulin resistance, impaired glucose tolerance, hypertension, etc.

Different models of MS have been described in the literature - genetic or diet-induced. The last are considered as better reflecting the condition in men, since it is the result mainly of the so called 'Western' pattern of feeding, characterized by the intake of animal fat high in saturated fatty acids, and fructose as a widely used sweetener, particularly in the form of the glucose-fructose syrup.

Weight gain is one of the typical features of MS. Dietary manipulations on experimental animals however, do not always lead to increase in body weight and in this respect the results in the literature vary widely. In our experimental setting, similarly to Nagai (18), Ai (19) and others, the final body weight did not differ between control and dietary manipulated groups. A possible explanation of this finding is the fact that experimental groups consumed less of the food enriched in fat and fructose, probably due to the higher caloric intake with these diets.

The central abdominal obesity is an important symptom of MS. To evaluate this parameter in the present study the retroperitoneal fat pad has been used as a component of the visceral adiposity. The fat cell store around the kidney was significantly increased in both diets. The absolute sizes of this adipose tissue as well as its ratios relative to the body weights (the fat index) are more than twice larger in the experimental groups compared to the control one. Similar results are reported for all the fat visceral localizations in the dietary models of MS $(18,19,20)$.

Dyslipidemia in MS is typically a combination of lipid changes comprising mainly high TG levels and low HDL-cholesterol. While overt hypercholesterolemia is not characteristic, changes are usually found in the LDL-cholesterol fractions with the increase of the small dense particles known to be more atherogenic.

In experimental models of MS the dyslipidemia is usually presented by high levels of TG. In our experiments the groups HF and HFHF had both significant increase in TG more than 2 and 3 times, respectively, compared to controls. Though these groups did not differ between each other, the higher levels in
HFHF rats can be linked to the well documented lipogenic effect of fructose $(20,21,18,22)$.

Dietary models of MS in rats published in literature vary significantly in respect to the levels of total and LDL-cholesterol. In some cases these lipid parameters are elevated - e.g. in diets rich in fructose (12), or in diets rich in fat and fructose $(21,23)$. In others, no changes are reported in analogous experimental settings (20). In our models we have definitely increased levels of total cholesterol in both dietary manipulated groups.

The changes reported for HDL-cholesterol also vary widely, with predominance of the negative results. De Castro et al. (12) found decrease of HDLcholesterol only in young rats (age of 4 weeks), but not in older animals (age of 12 weeks). Our results are in agreement with these data in respect to older rats, since our animals are in the same age range. Interestingly, in a former study of ours, where MS was induced by only $10 \%$ fructose in the drinking water, we found low levels of HDL and normal total cholesterol. This could be interpreted as an indication that in the present experiment the lack of change in HDL-cholesterol might be caused by the high total cholesterol.

The atherogenic indices TG/HDL-cholesterol and total cholesterol/HDL-cholesterol were also high in our dietary groups. These indices are used in clinical medicine in estimating cardiovascular risk of patients $(15,16)$. In the presence of other risk factors in experimental conditions, these could possibly be utilized also in a model of cardiovascular pathology.

Insulin resistance as a basic characteristic of MS can be studied in experimental conditions by different methods. Insulin-tolerance test is frequently utilized for this purpose $(8,19)$. It can reveal the impaired response to insulin due to decreased sensitivity of insulin receptors. In our experimental setting rats from both dietary manipulated groups had decreased effects of insulin as reflected by the higher glucose blood levels, measured at the three 30 minute intervals during an hour and a half. Although significantly different from the control group, the levels of blood sugar did not differ between the two experimental groups.

Another index of insulin resistance - hyperinsulinemia, was found only in the group of animals 
Silvia Gancheva, Maria Zhelyazkova-Savova, Bistra Galunska et al.

receiving food enriched in lard and fructose. This fact renders the corresponding model (HFHF) closer to the MS in humans, where the insulin resistance is compensated by higher levels of insulin. The negative results in the rats fed with only lard-enriched food could be explained with the lower amount of fructose that they were taking. It is known that dietary fructose, when in excess, can contribute via different mechanisms to insulin resistance and other metabolic disturbances in MS (22). The high consumption of saturated fatty acids is associated with decreased sensitivity to insulin $(19,24,25)$, but according to some authors (26), feeding with animal fats results in insulin resistance only under conditions of hypercaloric intake.

MS is frequently manifested clinically by nonalcoholic fatty liver disease (27). Many of the experimental models of MS are also associated with changes in liver that are similar to hepatic steatosis. Such findings have been reported as a result of feeding animals with fat-enriched diet $(8,13)$, or with diet combining fat and carbohydrates $(12,28)$, as well as fructose-treated rats $(7,22)$. In our experiment liver TG were elevated significantly in the HFHF group compared to the control one.

Although MS is mainly a metabolic disorder, this condition can be regarded also as a state of oxidative stress $(29,30)$. It has been even defined by some authors a component of MS (31). In general oxidative stress occurs when the production of reactive oxygen species surpasses the antioxidant capacity of the biological system. In MS and diabetes type 2 it is not only the high blood glucose that contributes to the generation of free radicals, but the free fatty acids are also involved. On its turn, oxidative stress appears to be a critical mechanism for the glucotoxicity, inducing both cellular insulin resistance and injury to beta cells (32). Markers of oxidative stress have been shown to be increased in many experimental models of MS $(5,7,13,23)$. In fructose rich diet oxidative stress has been manifested also by reduced antioxidant defense (33). In our models of MS we measured plasma TBARS as a marker of lipid peroxidation and found a significant increase in both HF and HFHF rats, showing that with the two dietary approaches oxidative stress is present.

\section{CONCLUSION}

In the present study of experimental MS we utilized two similar dietary approaches by loading the normal rat chow and drinking water with different ratios of animal fat (lard) and simple carbohydrate (fructose). Unlike most of the models, described in the literature, we used relatively small amounts of these additives, aiming to mimic closely the ,cafeteria diet', typical for the unhealthy nutritional habits of Western culture. Nevertheless, these diets were associated with significantly higher caloric intake. Both models that we studied reproduced most of the metabolic features of MS in humans - visceral obesity, dyslipidemia, insulin resistance as measured by the ITT. Markers of oxidative stress were elevated in the two experimental groups. HFHF group presented evidence of changes resembling the non-alcoholic fatty liver disease - higher TG hepatic content.

Indeed, with most of the parameters studied, the effects of both diets were similar. Yet, HFHF diet produced more pronounced changes. In addition, serum level of insulin was found to be increased only in the HFHF group, as were the liver TG levels. The advantages, manifested by the HFHF model, though small, made us consider that the dietary manipulation with animal fat and fructose added to the food of rats together with $10 \%$ fructose in the drinking water, is the preferred option for a model of MS in these animals. Based on these results, we can conclude that this model can be given priority in experimental studies of MS.

\section{REFERENCES}

1. The IDF consensus worldwide definition of the metabolic syndrome 2006

2. The DECODE Insulin Study Group Plasma insulin and cardiovascular mortality in non-diabetic European men and women: a meta-analysis of data from eleven prospective studies. Diabetologia. 2004;47:1245-56.

3. Carr DB, Utzschneider KM, Hull RL, Kodama K, Retzlaff BM, Brunzell JD,et al. Intra-abdominal fat is a major determinant of the National Cholesterol Education Program Adult Treatment Panel III criteria for the metabolic syndrome. Diabetes. 2004;53(8):2087-94.

4. Third report of the National Cholesterol Education Program (NCEP) expert panel on detection, eval- 
uation, and treatment of high blood cholesterol in adults (Adult Treatment Panel III). Final report. Circulation. 2002;106:3143-3421.

5. García ME, Marra CA, Rebolledo OR. Glycoxidative stress-induced damage on lipid profile in a fructose-enriched diet model of insulin resistance in rats. Archives of Physiology and Biochemistry. 2010;116(1):42-49.

6. Maiztegui B, Borelli MI, Raschia MA, Del Zotto $\mathrm{H}$, Gagliardino JJ. Islet adaptive changes to fructose-induced insulin resistance: b-cell mass, glucokinase, glucose metabolism, and insulin secretion. Journal of Endocrinology. 2009;200:139-149.

7. Castro MC, Massa ML, Del Zotto H, Gagliardino JJ, Francini F. Rat liver uncoupling protein 2: Changes induced by a fructose-rich diet . Life Sciences 2011; 89: 609-614

8. Buettner R, Parhofer KG, Woenckhaus M, Wrede CE, Kunz-Schughart LA, Schölmerich J, et al. Defining high-fat-diet rat models: metabolic and molecular effects of different fat types. Journal of Molecular Endocrinology. 2006;36:485-501.

9. Buettner R, Schölmerich J, Bollheimer LC. Highfat diets: modeling the metabolic disorders of human obesity in rodents. Obesity (Silver Spring). 2007;15:798-808.

10. Panchal SK, Poudyal H, Iyer A, Nazer R, Alam A, Diwan V, et al. High-carbohydrate high-fat dietinduced metabolic syndrome and cardiovascular remodeling in rats. J Cardiovasc Pharmacol. 2011;57(1):51-64.

11. Couturier K, Batandier C, Awada M, Hininger-Favier I, Canini F, Anderson RA, et al. Cinnamon improves insulin sensitivity and alters the body composition in an animal model of the metabolic syndrome. Archives of Biochemistry and Biophysics. 2010;50:158-161.

12. De Castro UG, dos Santos RA, Silva ME, de Lima WG, Campagnole-Santos MJ, Alzamora AC. Agedependent effect of high-fructose and high-fat diets on lipid metabolism and lipid accumulation in liver and kidney of rats. Lipids in Health and Disease. 2013;12:136.

13. Panchal SK, Poudyal H, Arumugam TV, Brown L. Rutin Attenuates Metabolic Changes, Nonalcoholic Steatohepatitis, and Cardiovascular Remodeling in High-Carbohydrate, High-Fat Diet-Fed Rats. The Journal of Nutrition. 2011;141:1062-1069.
14. Fluttert M, Dalm S, Oitzl MS. A refined method for sequential blood sampling by tail incision in rats Laboratory. Animals. 2000;34:372-378.

15. Lemieux I, Lamarche B, Couillard C, Pascot A, Cantin B, Bergeron J, et al. Total Cholesterol/HDL Cholesterol Ratio vs LDL Cholesterol/HDL Cholesterol Ratio as Indices of Ischemic Heart Dis-ease Risk in Men. Archives of Internal Medicine. 2001;161(22):2685-2692.

16. Dobiásova $\mathrm{M}$, Frohlichb J. The plasma parameter $\log$ (TG/HDL-C) as an atherogenic index: correlation with lipoprotein particle size and esterification rate in apob-lipoprotein-depleted plasma (FERHDL). Clinical Biochemistry. 2001;34(7):583-588.

17. Ohkawa H, Ohishi N, Yagi K. Assay for lipid peroxides in animal tissues by thiobarbituric acid reaction. Analytical Biochemistry. 1979;95(2):351-358.

18. Nagai Y, Yonemitsu S, Erion DM, Iwasaki T, Stark $\mathrm{R}$, Weismann D, et al. The Role of Peroxisome Pro-liferator-activated Receptor Gamma Coactivator 1 beta (PGC-1 $\beta$ ) in the Pathogenesis of Fructose-In-duced Insulin Resistance. Cell Metab. 2009; 9(3): 252-264.

19. Jing A, Ning W, Mei Y, Zhi-Min D, Yong-Chun Z, Bao-Feng Y. Development of Wistar rat mod-el of insulin resistance. World J Gastroenterol. 2005;11(24):3675-3679.

20. Mamikutty N, Thent ZC, Sapri SR, Sahruddin NN, Yusof MRM, Suhaimi FH. The Establish-ment of Metabolic Syndrome Model by Induction of Fructose Drinking Water in Male Wistar Rats. BioMed Research International. 2014; ID 263897; doi:10.1155/2014/263897

21. Panchal S, Brown L. Rodent Models for Met-abolic Syndrome Research. Journal of Bio-medicine and Biotechnology. 2011;ID 351982; doi:10.1155/2011/351982

22. Dekker MJ, Su Q, Baker C, Rutledge AC, Adeli K. Fructose: a highly lipogenic nutrient implicated in insulin resistance, hepatic steatosis, and the metabolic syndrome. Am J Physiol Endocrinol Metab. 2010; 299(5): 685-694.

23. Alam A, Kauter K, Brown L. Naringin Improves Diet-Induced Cardiovascular Dysfunction and Obesity in High Carbohydrate, High Fat Diet-Fed Rats. Nutrients. 2013;5:637-650.

24. Roden M, Price TB, Perseghin G, Petersen KF, Rothman DL, Cline GW, et al. Mechanism of free 
fatty acid-induced insulin resistance in humans. J

Clin Invest. 1996;97(12):2859-2865.

25. Funaki M. Saturated fatty acids and insulin resistance. J. Med. Invest. 2009;56:88-92.

26. Cordain L, Eades MR, Eades MD. Hyperinsulinemic diseases of civilization: more than just Syndrome X. Comp Biochem Physiol A Mol Integr Physiol. 2003;136(1):95-112.

27. Adams LA, Angulo P, Lindor KD. Nonalcoholic fatty liver disease. Canadian Medical Association Journal. 2005;172(7):899-905.

28. Basaranoglu M, Basaranoglu G, Sabuncu T, Sentürk H. Fructose as a key player in the development of fatty liver disease. World J Gastroenterol. 2013; 19(8): 1166-1172.

29. Ando K, Fujita T. Metabolic syndrome and oxidative stress. Free Radical Biology \& Medicine. 2009;47:213-218.

30. Roberts CK, Sindhu KK. Oxidative stress and metabolic syndrome. Life Sciences 2009;84:705-712.

31. Hopps E, Noto D, Caimi G, Averna MR. A novel component of the metabolic syndrome: The oxidative stress. Nutrition, Metabolism and Cardiovascular Diseases 2010;20(1):72-77.

32. Eriksson JW. Metabolic stress in insulin's target cells leads to ROS accumulation - A hypothetical common pathway causing insulin resistance. EFBS Letters 2007;581(19):3734-3742.

33. Girard A, Madani S, Boukortt F, Cherkaoui-Malki M, Belleville J, Prost J. Fructose-enriched diet modifies antioxidant status and lipid metabolism in spontaneously hypertensive rats. Nutrition. 2006;22:758-766. 